\title{
(1) Mortality risks associated with emergency admissions during weekends and public holidays: an analysis of electronic health records
}

oa

Lancet 2017; 390: 62-72 Published Online May 9, 2017

http://dx.doi.org/10.1016/

S0140-6736(17)30782-1

See Comment page 8

*Contributed equally

Oxford Biomedical Research

Centre (Prof A S Walker PhD,

A Mason DPhil, T P Quan MSc

NJ Fawcett MBChB

PWatkinson MD,

Prof M Llewelyn PhD,

N Stoesser DPhil, J Finney MSc,

Prof J Davies PhD

D H Wyllie DPhil,

Prof D W Crook MBBCh,

ProfTE A Peto DPhil), Nuffield

Department of Medicine, University of Oxford

(Prof A S Walker, A Mason,

T P Quan, NJ Fawcett, Prof M Llewelyn, N Stoesser, J Finney, D H Wyllie, Prof D W (rook, ProfT EA Peto),

Oxford University Hospitals

NHS Foundation Trust

(N) Fawcett, P Watkinson,

N Stoesser, Prof D W Crook,

ProfT E A Peto), and Nuffield

Department of Clinical

Neurosciences (P Watkinson), John Radcliffe Hospital, Oxford

UK; Brighton and Sussex Medical School, University of Sussex, Falmer, UK

(Prof M Llewelyn); Department of Computer Science,

University of Oxford, Oxford, UK (Prof J Davies); and National Infection Service, Public Health England, Colindale, London, UK

(D H Wyllie, Prof D W Crook)

Correspondence to: Prof A Sarah Walker, Microbiology Level 7, John Radcliffe Hospital, Oxford OX3 9DU, UK

sarah.walker@ndm.ox.ac.uk

See Online for appendix

A Sarah Walker*, Amy Mason*, T Phuong Quan, Nicola J Fawcett, Peter Watkinson, Martin Llewelyn, Nicole Stoesser, John Finney, Jim Davies,

David H Wyllie, Derrick W Crook, Tim E A Peto

Summary

Background Weekend hospital admission is associated with increased mortality, but the contributions of varying illness severity and admission time to this weekend effect remain unexplored.

Methods We analysed unselected emergency admissions to four Oxford University National Health Service hospitals in the UK from Jan 1, 2006, to Dec 31, 2014. The primary outcome was death within 30 days of admission (in or out of hospital), analysed using Cox models measuring time from admission. The primary exposure was day of the week of admission. We adjusted for multiple confounders including demographics, comorbidities, and admission characteristics, incorporating non-linearity and interactions. Models then considered the effect of adjusting for 15 common haematology and biochemistry test results or proxies for hospital workload.

Findings 257596 individuals underwent 503938 emergency admissions. 18313 (4.7\%) patients admitted as weekday energency admissions and $6070(5 \cdot 1 \%)$ patients admitted as weekend emergency admissions died within 30 days $(\mathrm{p}<0 \cdot 0001) .9347$ individuals underwent 9707 emergency admissions on public holidays. 559 (5.8\%) died within 30 days $(\mathbf{p}<0.0001 v s$ weekday). 15 routine haematology and biochemistry test results were highly prognostic for mortality. In 271465 (53 . 9\%) admissions with complete data, adjustment for test results explained 33\% (95\% CI 21 to 70 ) of the excess mortality associated with emergency admission on Saturdays compared with Wednesdays, $52 \%$ (lower 95\% CI 34) on Sundays, and $87 \%$ (lower $95 \%$ CI 45 ) on public holidays after adjustment for standard patient characteristics. Excess mortality was predominantly restricted to admissions between $1100 \mathrm{~h}$ and $1500 \mathrm{~h}\left(\mathrm{p}_{\text {interaction }}=0.04\right)$. No hospital workload measure was independently associated with mortality (all $p$ values $>0 \cdot 06$ ).

Interpretation Adjustment for routine test results substantially reduced excess mortality associated with emergency admission at weekends and public holidays. Adjustment for patient-level factors not available in our study might further reduce the residual excess mortality, particularly as this clustered around midday at weekends. Hospital workload was not associated with mortality. Together, these findings suggest that the weekend effect arises from patient-level differences at admission rather than reduced hospital staffing or services.

\section{Funding NIHR Oxford Biomedical Research Centre.}

Copyright (C) The Author(s). Published by Elsevier Ltd. This is an Open Access article under the CC BY-NC-ND 4.0 license.

\section{Introduction}

Substantial medical and public attention has been paid to the so-called weekend effect, namely that patients admitted to hospital at the weekend have greater mortality than do patients admitted on weekdays. ${ }^{1}$ This independent effect has been demonstrated in large national and international studies, in elective, ${ }^{2}$ emergency ${ }^{3-6}$ and all admissions..$^{7-10}$

Although the excess mortality risk associated with weekend admission appears clear, the underlying causal mechanisms are not. Many hospitals are widely assumed to have fewer staff and resources at weekends than on weekdays, affecting patient care. ${ }^{11}$ Findings from studies into the weekend effect have led the UK Government to commit to providing National Health Service (NHS) hospital care as a 7-day service. ${ }^{12,13}$

However, it is being admitted at weekends, rather than merely being in hospital at weekends, that has been consistently associated with higher mortality risk, reduced weekend staffing and resources should affect all patients in hospital at weekends, not just those newly admitted. Alternative explanations for the weekend effect include variation in patient factors (eg, presenting illness severity, delayed presentation, frailty) that are not completely captured in administrative datasets used in many studies to date. In such studies ${ }^{1,2}$ the factor most closely reflective of the severity of presenting illness is the intrinsic mortality risk score based on clinical classifications software, ${ }^{14}$ which leaves substantial potential for residual confounding of admission-day effects by the individual patient's illness severity (appendix p 24). Findings from a 2016 study $^{15}$ showing increased mortality risk only in patients admitted at weekends from general practitioners (rather than via the emergency room) provide indirect evidence supporting this notion. 
Research in context

\section{Evidence before this study}

We searched PubMed for publications from inception up until June 15, 2016, with the terms "weekend" AND "admission" AND "mortality", with no language restrictions, and also reviewed references from retrieved articles. Weekend emergency admissions have been associated with an excess risk of dying in the next 30 days compared with weekday admissions (after adjusting for patient-level factors available in administrative datasets) in large national and international studies, in all admissions and in subgroups defined by elective admissions, emergency admissions, and clinical subgroups (eg, stroke). The excess mortality is often attributed to differences in staffing levels or service provision at the weekends. However, there is indirect evidence that the excess mortality could be attributed to inadequate adjustment for how sick the patients admitted at weekends are.

\section{Added value of this study}

We used a large comprehensive warehouse of electronic health records to adjust individual-level analyses for factors not previously considered, including haematology, biochemistry, and microbiology tests, and time of admission. We found that adjustment for 15 routinely measured haematology and biochemistry test results could explain a substantial proportion of the excess mortality effect associated with weekend emergency admission. The remaining excess deaths predominantly occurred in patients admitted as emergencies between $1100 \mathrm{~h}$ and $1500 \mathrm{~h}$ at the weekend. The excess mortality was not associated with multiple measures of hospital workload.

\section{Implications of all available evidence}

These results are not consistent with staffing or service provision being the primary driver of the weekend effect, and suggest that much of the remaining excess mortality could be accounted for by other characteristics of the patients, their health-seeking behaviour, or availability of services outside the hospital.

specific surgery lists to particular weekdays). IORD has Research Ethics Committee and Health Research Authority approval (14/SC/1069, ECC5-07[a]/2009).

Studies using clinical datasets with more detailed prognostic data have generally been restricted to specific conditions ${ }^{16}$ or have been much smaller. It is also unclear how the excess risk is distributed across the $48 \mathrm{~h}$ of weekend admissions (with time of admission unavailable in hospital episode statistics). Here, we use information from common haematology and biochemistry tests done around admission, and admission time, from the Infections in Oxfordshire Research Database (IORD) ${ }^{17}$ to investigate explanations for the mortality weekend effect in emergency admissions to one large hospital group.

\section{Methods}

\section{Study design and participants}

IORD contains anonymised data from all admissions to the four hospitals that make up the Oxford University Hospitals NHS Foundation Trust from April 1, 1997, linked to haematology, biochemistry, and microbiology test results. ${ }^{17}$ These hospitals (which include a district general hospital, a large teaching hospital, a specialist orthopaedic hospital, and a major cancer centre) provide all acute care and pathology services in the region, supplying a population of about 600000 individuals. Out-of-hospital mortality is determined by regular updates from the national information system that records all UK deaths, the National Health Service clinical spine application.

We analysed data from all emergency admissions (defined by all codes with prefix 2 for the variable "admission method"), including patients admitted via the accident and emergency department, general practitioners, consultant clinics, and other methods from Jan 1, 2006, to Dec 31, 2014. We excluded elective admissions because different factors might affect risks associated with weekend admission (eg, restriction of

\section{Outcomes}

The primary outcome was 30-day mortality (in hospital or after discharge) for consistency with previous studies, censoring at the earliest of the following three dates: 30 days after admission, last routine vital status update, or last subsequent admission or discharge. Cox regression (which implicitly adjusts for days at risk) considered days from admission.

\section{Statistical analysis}

As exposures, we first considered all admission factors adjusted for in previous NHS studies (appendix p 24). ${ }^{1,2}$ Of 504563 total emergency admissions, we excluded 69 admissions with missing age, one with negative age, seven admissions with missing sex or intersex, and 548 admissions with missing diagnostic codes (total $625[0 \cdot 1 \%])$, leaving 503938 admissions in regression analyses. The primary exposure was admission day of the week, prespecified to be included irrespective of statistical significance; as in previous studies, ${ }^{1,2}$ the reference category was Wednesday because this is the middle of the week. For other factors, model selection used backwards elimination based on minimisation of the Bayesian information criterion, using natural cubic splines for non-linear effects in continuous factors. We then identified pairwise interactions between included factors using forward selection based on the Bayesian information criterion to produce the final model (model A), also fitted to 7-day, 14-day, and 21-day mortality as secondary outcomes. Admission day of week was categorised as weekend (Saturday or Sunday) versus
For the National Health Service clinical spine application see https://digital.nhs.uk/spine 
weekday to increase power to identify interactions. Secondary analyses investigated mortality risk for admission on public holidays. Sensitivity analyses included current day of the week at risk in a timeupdated Cox model. Full details are provided in the appendix (p 4).

We then investigated the subgroup with 15 commonly performed haematology and biochemistry test results (haemoglobin, platelets, lymphocytes, neutrophils, eosinophils, monocytes, C-reactive protein, urea, bilirubin, creatinine, albumin, alanine aminotransferase, alkaline phosphatase, sodium, and potassium, using the closest result to admission time within 2 calendar days before or after admission). Several of these tests reflect the presence of underlying infection. We chose tests on the basis of the percentage of emergency admissions in whom they were complete (appendix p 6). We first confirmed that model A provided similar results in all emergency admissions and in this subgroup, and then selected additional predictors from the 15 test results as previously described (model B), incorporating non-linearity with natural cubic splines. We did not do multiple imputation because it would have required development of models for each test of similar complexity to model A (which included ten interactions and several non-linear effects), and could have increased rather than decreased bias if these models were mis-specified..$^{18}$ We calculated $95 \%$ CIs for the proportion of the admission-day effect explained by these test results (log scale) using Fieller's method, ${ }^{19}$ reporting only lower limits when upper limits exceeded $100 \%$. We estimated excess hazards associated with admission day over time from admission using flexible parametric models. ${ }^{20}$

To estimate the effect of hospital workload, we used three proxy measures (total admissions, total net hospital occupancy [admissions minus discharges], and percentage of bed occupancy based on inpatient duration); staffing information was not available. If the weekend effect were due to understaffing or lack of services, increased mortality would also be expected when the hospital was fuller than average for any given day of the week, because rotas are fixed in advance, with extra staff only called in for major incidents. We therefore normalised our three proxy measures, calculated for each calendar day, to the typical value for each day of the week for each calendar year (appendix p 8).

We undertook additional sensitivity analyses to assess the robustness of our findings (appendix $p$ 7). In exploratory analyses, we calculated the unadjusted and adjusted mortality differences by hour of admission at weekends versus weekdays. Admission hour was then split into a four-level categorical variable at $0800 \mathrm{~h}$, 1100 h, 1500 h, and 0000 h (consistent with Freemantle and colleagues ${ }^{7}$. $p$ values are two-sided and tested the null hypothesis of no effect. We used Stata version 14.1 for all analyses.

\section{Role of the funding source}

The funder had no role in study design, data collection, analysis, or writing of the report. The corresponding author had full access to the study data and final responsibility for the decision to submit for publication.

\section{Results}

Analyses included 503938 emergency admissions from 2006-14 for 257596 individuals. Substantially fewer emergency admissions occurred at weekends than on weekdays (figure 1). Admissions increased year on year, but weekend and weekday patterns remained consistent over the years (figure 1). Charlson score and age were similar in weekend and weekday admissions (figure 1).

$24383(4 \cdot 8 \%)$ emergency admissions resulted in death within 30 days of admission (in hospital or after discharge). $18313(4.7 \%)$ of 385647 patients admitted on a weekday died within 30 days of admission, compared with $6070(5 \cdot 1 \%)$ of 118291 patients admitted on a weekend $(\mathrm{p}<0.0001$; table, appendix pp 12, 25). Median length of stay was 2 calendar days (IQR 1-6). Most time at risk during the 30 days of follow-up was therefore spent outside hospital. 271465 (53.9\%) admissions had all 15 haematology and biochemistry results, ranging from $53.0 \%$ to $54.5 \%$ across admission days (18244 [74.8\%] of 24383 admissions after which patients died within 30 days vs 253221 [52.8\%] of 479555 admissions after which patients were not known to have died within 30 days; appendix p 6).

Using model $A$, all the factors adjusted for in previous published analyses or studies of administrative datasets had an independent effect on 30-day mortality (C-statistic 0.88; appendix pp 9, 12), as did weekend emergency admission (adjusted relative risk [aRR] for Saturday vs Wednesday 1.08 [95\% CI 1.03-1.14], aRR for Sunday vs Wednesday 1.09 [1.03-1.14]; figure 2). This excess mortality was slightly larger when we fitted the identical model to emergency admissions with all 15 test results (aRR for Saturday vs Wednesday 1.11 [95\% CI 1.05-1.17], aRR for Sunday vs Wednesday 1·11 [1.05-1.18]; C-statistic 0.84; figure 2, appendix p 12), but we found no evidence of heterogeneity $\left(p_{\text {interaction }}=0 \cdot 18\right)$. There was no evidence that admissions with complete test results had higher adjusted mortality than those without all test results (aRR 1.01 [95\% CI 0.98-1.04], $\mathrm{p}=0 \cdot 40$ ). Absolute mortality risks and excess risks associated with weekend admission, were greatest in the first 2-5 days after admission (figure 3).

Values for nine of the 15 test results were markedly different for weekend versus weekday emergency admissions (eg, median neutrophil count and C-reactive protein; figure 1, appendix p 26). This difference was predominantly due to proportionately fewer patients with normal results being admitted at weekends than on weekdays, although the absolute numbers of 

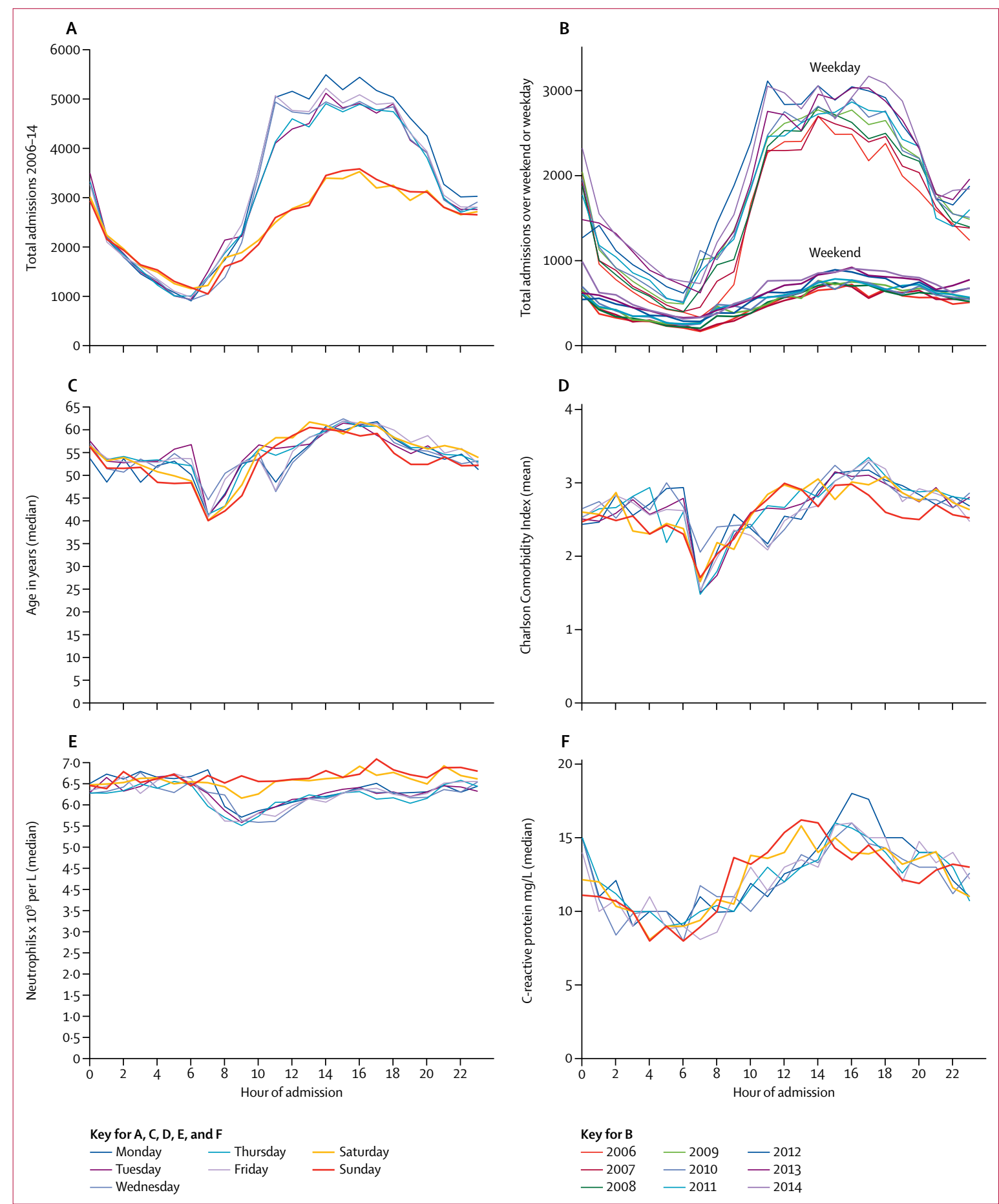

Figure 1: Characteristics of emergency admissions

(A) Mean total number of admissions over 8 years by day of the week. (B) Total number of admissions by calendar year and weekday vs weekend. (C) Median age at admission. (D) Mean Charlson Comorbidity Index (68.6\% of admissions had Charlson score 0 , so mean rather than median is shown). (E) Median neutrophils at admission ( $\left.\times 10^{9} / \mathrm{L}\right)$. (F) Median C-reactive protein concentration at admission ( $\left.\mathrm{mg} / \mathrm{L}\right)$.

admissions with abnormal results also fell (appendix p 27-28). In model $\mathrm{B}$, each test result independently predicted 30-day mortality after adjustment for model A factors (appendix p 30). Several test results, notably lymphocyte count and sodium and urea concentrations, affected mortality risk even within normal ranges. Eight interactions with test results added predictive information (C-statistic 0 89; appendix pp 10, 30). 
In admissions with complete data, test results accounted for 33\% (95\% CI 21 to 70 ) of the excess mortality risk for emergency admission on Saturday and 52\% (lower 95\% CI 34) of the risk for Sunday, compared with emergency admission on Wednesday (figure 2). The adjusted relative risk for residual excess mortality (ie, after adjusting for test results) fell to 1.07 (95\% CI $1 \cdot 01-1 \cdot 13)$ for Saturday and 1.05 (1.00-1.11) for Sunday, compared with Wednesday. The residual excess mortality associated with weekend emergency admission was not restricted to any patient or clinical subgroup defined by model A factors, and did not vary by calendar year

\begin{tabular}{|c|c|c|c|c|c|}
\hline & $\begin{array}{l}\text { Emergency } \\
\text { admissions } \\
(n=503938)\end{array}$ & $\begin{array}{l}\text { Emergency admissions } \\
\text { on weekdays } \\
(n=385647)\end{array}$ & $\begin{array}{l}\text { Emergency } \\
\text { admissions on } \\
\text { weekends ( } n=118291)\end{array}$ & $\begin{array}{l}\text { Deaths within } \\
30 \text { days }(n=24383)^{*}\end{array}$ & $\begin{array}{l}\text { Emergency admissions } \\
\text { with complete test } \\
\text { results }(n=271465)\end{array}$ \\
\hline \multicolumn{6}{|l|}{ Day of admission } \\
\hline Monday & 80950 (16.1\%) & .. & .. & $3900(4 \cdot 8 \%)$ & $43662(16 \cdot 1 \%)$ \\
\hline Tuesday & $75596(15 \cdot 0 \%)$ &.. & .. & $3611(4 \cdot 8 \%)$ & $41101(15 \cdot 1 \%)$ \\
\hline Wednesday & $75732(15 \cdot 0 \%)$ &. & .. & $3607(4 \cdot 8 \%)$ & $41059(15 \cdot 1 \%)$ \\
\hline Thursday & $74975(14 \cdot 9 \%)$ &.$\cdot$ & .. & $3541(4 \cdot 7 \%)$ & $40867(15 \cdot 1 \%)$ \\
\hline Friday & $78394(15 \cdot 6 \%)$ &.$\cdot$ & .. & $3654(4 \cdot 7 \%)$ & $41579(15 \cdot 3 \%)$ \\
\hline Saturday & $59242(11.8 \%)$ & .. & .. & $3100(5 \cdot 2 \%)$ & $31469(11.6 \%)$ \\
\hline Sunday & $59049(11 \cdot 7 \%)$ & .. &.$\cdot$ & $2970(5 \cdot 0 \%)$ & $31728(11 \cdot 7 \%)$ \\
\hline Calendar year & $2010(2008-2013)$ & $2010(2008-2012)$ & 2010 (2008-2013) & $2010(2008-2012)$ & $2010(2008-2013)$ \\
\hline \multicolumn{6}{|l|}{ Admission method } \\
\hline Accident and emergency & $268193(53 \cdot 2 \%)$ & $188189(48 \cdot 8 \%)$ & $80004(67 \cdot 6 \%)$ & $12085(4 \cdot 5 \%)$ & $135893(50 \cdot 1 \%)$ \\
\hline Consultant clinic & $28506(5 \cdot 7 \%)$ & $26799(6 \cdot 9 \%)$ & $1707(1.4 \%)$ & $603(2 \cdot 1 \%)$ & $8515(3 \cdot 1 \%)$ \\
\hline General practitioner & $134521(26 \cdot 7 \%)$ & $113655(29 \cdot 5 \%)$ & $20866(17 \cdot 6 \%)$ & $8813(6 \cdot 6 \%)$ & $92846(34 \cdot 2 \%)$ \\
\hline Other & $72718(14 \cdot 4 \%)$ & $57004(14.8 \%)$ & $15714(13 \cdot 3 \%)$ & $2882(4 \cdot 0 \%)$ & $34211(12 \cdot 6 \%)$ \\
\hline \multicolumn{6}{|l|}{ Admission source } \\
\hline Other NHS hospitals & $9181(1 \cdot 8 \%)$ & $6786(1.8 \%)$ & $2395(2 \cdot 0 \%)$ & $535(5 \cdot 8 \%)$ & $5558(2 \cdot 0 \%)$ \\
\hline Other & $2860(0.6 \%)$ & $2272(0 \cdot 6 \%)$ & $588(0 \cdot 5 \%)$ & $376(13 \cdot 1 \%)$ & $1782(0 \cdot 7 \%)$ \\
\hline $\begin{array}{l}\text { Temporary place of } \\
\text { residence }\end{array}$ & $3723(0 \cdot 7 \%)$ & $2681(0 \cdot 7 \%)$ & $1042(0 \cdot 9 \%)$ & $148(4 \cdot 0 \%)$ & $2120(0 \cdot 8 \%)$ \\
\hline Usual place of residence & $488174(96 \cdot 9 \%)$ & $373908(97 \cdot 0 \%)$ & $114266(96 \cdot 6 \%)$ & $23324(4 \cdot 8 \%)$ & $262005(96 \cdot 5 \%)$ \\
\hline \multicolumn{6}{|l|}{ Admission specialty } \\
\hline Medical & $286705(56 \cdot 9 \%)$ & $224050(58 \cdot 1 \%)$ & $62655(53 \cdot 0 \%)$ & $19542(6 \cdot 8 \%)$ & $173117(63 \cdot 8 \%)$ \\
\hline Surgical & $205289(40 \cdot 7 \%)$ & $152430(39 \cdot 5 \%)$ & $52859(44.7 \%)$ & $4272(2 \cdot 1 \%)$ & $94903(35 \cdot 0 \%)$ \\
\hline Other & $11944(2 \cdot 4 \%)$ & $9167(2 \cdot 4 \%)$ & $2777(2 \cdot 3 \%)$ & $569(4 \cdot 8 \%)$ & $3445(1 \cdot 3 \%)$ \\
\hline Charlson Comorbidity Index & $0(0-4)$ & $0(0-4)$ & $0(0-4)$ & $10(0-14)$ & $0(0-7)$ \\
\hline $\begin{array}{l}\text { Number of admissions in } \\
\text { the past year }\end{array}$ & $0(0-2)$ & $0(0-2)$ & $0(0-2)$ & $1(0-3)$ & $1(0-2)$ \\
\hline $\begin{array}{l}\text { Any complex admissions in } \\
\text { the past year }\end{array}$ & $56491(11 \cdot 2 \%)$ & $43250(11 \cdot 2 \%)$ & $13241(11 \cdot 2 \%)$ & $5555(9 \cdot 8 \%)$ & $38589(14 \cdot 2 \%)$ \\
\hline \multicolumn{6}{|c|}{ Intrinsic risk quintile (appendix p 4) } \\
\hline 1 (lowest risk) & $21379(4 \cdot 2 \%)$ & $17747(4 \cdot 6 \%)$ & $3632(3 \cdot 1 \%)$ & $9(<0 \cdot 1 \%)$ & $5184(1 \cdot 9 \%)$ \\
\hline 2 & $57272(11 \cdot 4 \%)$ & $44271(11 \cdot 5 \%)$ & $13001(11 \cdot 0 \%)$ & $139(0 \cdot 2 \%)$ & $20679(7.6 \%)$ \\
\hline 3 & $115507(22 \cdot 9 \%)$ & $87519(22 \cdot 7 \%)$ & $27988(23 \cdot 7 \%)$ & $931(0.8 \%)$ & $51448(19 \cdot 0 \%)$ \\
\hline 4 & $135055(26 \cdot 8 \%)$ & $102623(26 \cdot 6 \%)$ & $32432(27 \cdot 4 \%)$ & $3152(2 \cdot 3 \%)$ & $73960(27 \cdot 2 \%)$ \\
\hline 5 (highest risk) & $174725(34 \cdot 7 \%)$ & $133487(34 \cdot 6 \%)$ & $41238(34 \cdot 9 \%)$ & $20152(11 \cdot 5 \%)$ & $120194(44 \cdot 3 \%)$ \\
\hline Age at last birthday, years & $55(29-76)$ & $55(30-76)$ & $55(27-77)$ & $80(69-87)$ & $64(42-79)$ \\
\hline \multicolumn{6}{|l|}{ Sex } \\
\hline Female & $255330(50 \cdot 7 \%)$ & $196295(50 \cdot 9 \%)$ & 59035 (49.9\%) & $11918(4 \cdot 7 \%)$ & $140123(51 \cdot 6 \%)$ \\
\hline Male & $248608(49 \cdot 3 \%)$ & $189352(49 \cdot 1 \%)$ & $59256(50 \cdot 1 \%)$ & $12465(5 \cdot 0 \%)$ & $131342(48 \cdot 4 \%)$ \\
\hline \multicolumn{6}{|l|}{ Ethnicity } \\
\hline White & $410814(81 \cdot 5 \%)$ & $316746(82 \cdot 1 \%)$ & $94068(79 \cdot 5 \%)$ & $21472(5 \cdot 2 \%)$ & $228714(84 \cdot 3 \%)$ \\
\hline Black & $5501(1 \cdot 1 \%)$ & $4258(1 \cdot 1 \%)$ & $1243(1 \cdot 1 \%)$ & $90(1 \cdot 6 \%)$ & $2657(1 \cdot 0 \%)$ \\
\hline Asian & $14347(2 \cdot 8 \%)$ & $11018(2 \cdot 9 \%)$ & $3329(2 \cdot 8 \%)$ & $221(1 \cdot 5 \%)$ & $6637(2 \cdot 4 \%)$ \\
\hline Other & $9386(1 \cdot 9 \%)$ & $7075(1 \cdot 8 \%)$ & $2311(2 \cdot 0 \%)$ & $148(1 \cdot 6 \%)$ & $3927(1 \cdot 4 \%)$ \\
\hline \multirow[t]{2}{*}{ Unknown } & $63890(12 \cdot 7 \%)$ & $46550(12 \cdot 1 \%)$ & $17340(14 \cdot 7 \%)$ & $2452(3 \cdot 8 \%)$ & $29530(10 \cdot 9 \%)$ \\
\hline & & & & \multicolumn{2}{|c|}{ (Table continues on next page } \\
\hline
\end{tabular}




\begin{tabular}{|c|c|c|c|c|c|}
\hline & $\begin{array}{l}\text { Emergency } \\
\text { admissions } \\
(n=503938)\end{array}$ & $\begin{array}{l}\text { Emergency admissions } \\
\text { on weekdays } \\
(\mathrm{n}=385647)\end{array}$ & $\begin{array}{l}\text { Emergency } \\
\text { admissions on } \\
\text { weekends }(n=118291)\end{array}$ & $\begin{array}{l}\text { Deaths within } \\
30 \text { days }(n=24383)^{*}\end{array}$ & $\begin{array}{l}\text { Emergency admissions } \\
\text { with complete test } \\
\text { results ( } n=271465)\end{array}$ \\
\hline \multicolumn{6}{|c|}{ (Continued from previous page) } \\
\hline \multicolumn{6}{|c|}{ Ten most prevalent admission diagnoses or diagnosis groups according to clinical classifications software } \\
\hline Other & $127254(25 \cdot 3 \%)$ & $99668(25 \cdot 8 \%)$ & $27586(23 \cdot 3 \%)$ & $10850(8 \cdot 5 \%)$ & $77521(28.6 \%)$ \\
\hline Low-risk & $85321(16 \cdot 9 \%)$ & $66260(17 \cdot 2 \%)$ & $19061(16 \cdot 1 \%)$ & $203(0.2 \%)$ & $30580(11 \cdot 3 \%)$ \\
\hline Non-specific chest pain & $20802(4 \cdot 1 \%)$ & $16176(4 \cdot 2 \%)$ & $4626(3 \cdot 9 \%)$ & $119(0 \cdot 6 \%)$ & $11103(4 \cdot 1 \%)$ \\
\hline Abdominal pain & $18486(3 \cdot 7 \%)$ & $14392(3.7 \%)$ & $4094(3 \cdot 5 \%)$ & $158(0 \cdot 9 \%)$ & $13659(5 \cdot 0 \%)$ \\
\hline Pneumonia & $12582(2 \cdot 5 \%)$ & $9336(2 \cdot 4 \%)$ & $3246(2 \cdot 7 \%)$ & $2567(20 \cdot 4 \%)$ & $10109(3.7 \%)$ \\
\hline Acute bronchitis & $11675(2 \cdot 3 \%)$ & $8809(2 \cdot 3 \%)$ & $2866(2 \cdot 4 \%)$ & $771(6 \cdot 6 \%)$ & $6759(2.5 \%)$ \\
\hline $\begin{array}{l}\text { Superficial injury, } \\
\text { contusion }\end{array}$ & $11236(2 \cdot 2 \%)$ & $7737(2 \cdot 0 \%)$ & $3499(3.0 \%)$ & $173(1 \cdot 5 \%)$ & $2801(1 \cdot 0 \%)$ \\
\hline Urinary tract infection & $11182(2 \cdot 2 \%)$ & $8218(2 \cdot 1 \%)$ & $2964(2 \cdot 5 \%)$ & $571(5 \cdot 1 \%)$ & $8622(3 \cdot 2 \%)$ \\
\hline Fracture of upper limb & $10194(2 \cdot 0 \%)$ & $7347(1 \cdot 9 \%)$ & $2847(2 \cdot 4 \%)$ & $87(0.9 \%)$ & $1354(0 \cdot 5 \%)$ \\
\hline $\begin{array}{l}\text { Skin and subcutaneous } \\
\text { tissue infection }\end{array}$ & $9874(2 \cdot 0 \%)$ & $7750(2 \cdot 0 \%)$ & $2124(1 \cdot 8 \%)$ & $127(1 \cdot 3 \%)$ & $6223(2 \cdot 3 \%)$ \\
\hline \multicolumn{6}{|c|}{$\begin{array}{l}\text { Data are }(\%) \text { or median (IQR). Details about other categories, other clinical classification software groups, and relative risk estimates from unadjusted and adjusted models } \\
\text { are provided in the appendix (p 12). NHS=National Health Service. }{ }^{*} \text { Column shows number of deaths as a proportion of emergency admissions. }\end{array}$} \\
\hline
\end{tabular}

$\left(\mathrm{p}_{\text {interaction }}>0 \cdot 05\right.$; appendix $\mathrm{p}$ 19). Interactions between weekend versus weekday admission and alanine aminotransferase $\quad\left(\mathrm{p}_{\text {interaction }}=0.004\right)$ and bilirubin $\left(\mathrm{p}_{\text {interaction }}=0.002\right)$ were small in magnitude and did not explain the weekend effect (appendix p 40; $\mathrm{p}_{\text {interaction }}>0 \cdot 10$ for other interactions between weekend versus weekday admission and test results).

In sensitivity analyses, the addition of an indicator that blood had been taken for culture (suggesting doctors suspected bacterial infection) or for blood gas analysis improved model B ( $\mathrm{p}=0.02$ for blood culture and $\mathrm{p}<0.0001$ for blood gases), but did not affect excess mortality risk associated with weekend emergency admission (figure 2). Time since last inpatient admission and duration of last inpatient admission were highly prognostic in addition to model A and model B (data not shown), but did not alter excess mortality risk associated with weekend admission (appendix p 20).

63961 (12.7\%) admissions in 37119 (14.4\%) individuals were within 30 days of a previous admission. Exclusion of these readmissions did not affect results (appendix p 20). Other sensitivity analyses (appendix p 20) were also consistent with main analyses, including adjustment for current day of the week at risk as a time-updated factor (as well as admission day of the week; figure 2, appendix p 21). Similar excess risks were also associated with weekend emergency admission for 7-day, 14-day, and 21-day mortality (appendix p 42).

There were 73 non-weekend public holidays (typically Mondays) from 2006 to 2014. 9347 (3.6\%) individuals underwent 9707 (1.9\%) emergency admissions on public holidays; $559(5 \cdot 8 \%)$ of these admissions died within 30 days ( $<<0.0001$ vs weekday). Compared with weekdays, excess mortality risk was similar for emergency admissions on public holidays and weekends, both unadjusted and adjusted for model A factors (figure 2). Adjustment for haematology and biochemistry test results accounted for $87 \%$ (lower $95 \%$ CI 45 ) of the excess risk associated with emergency admission on public holidays (compared with Wednesdays).

Proxy measures for workload showed increasing pressure on hospital resources over time (appendix p 43). No measure of hospital workload on specific calendar days, relative to that expected for that day of the week in that calendar year, was independently associated with 30-day mortality (model A, p >0.06; model B, $\mathrm{p}>0 \cdot 16$; appendix p 22). Adjustment for these measures did not attenuate the effect of admission day of the week (appendix p 47).

We noted fewer emergency admissions from $1000 \mathrm{~h}$ to $2200 \mathrm{~h}$ at weekends than on weekdays, but similar numbers from $2200 \mathrm{~h}$ to $1000 \mathrm{~h}$ (figure 1). Mortality risk differed significantly by admission hour, being lowest at 0800-1100 h (figure 4). The largest differences in mortality risk between weekend and weekdays were between $1100 \mathrm{~h}$ and $1500 \mathrm{~h}$. Circadian variation in mortality risk was as great as (if not greater than) differences in risk between weekends and weekdays. The addition of categorised admission hour significantly improved both model A and model B $(\mathrm{p}<0 \cdot 0001)$. The weekend versus weekday effect depended on time of day of admission in model B $\left(\mathrm{p}_{\text {interaction }}=0.04\right.$; model A $\mathrm{p}_{\text {interaction }}=0 \cdot 11$; figure 4). After adjustment for model A factors, excess mortality risks associated with weekend admission were somewhat greater for admissions between $0800 \mathrm{~h}$ and $0000 \mathrm{~h}$, and somewhat less for admissions between $0000 \mathrm{~h}$ and $0800 \mathrm{~h} \mathrm{(} \mathrm{p}=0.07$; figure 4), with the largest excess for admissions between $1100 \mathrm{~h}$ and $1500 \mathrm{~h}(\mathrm{p}<0 \cdot 0001)$. Adjusting for test results in model B significantly reduced the weekend excess risk 


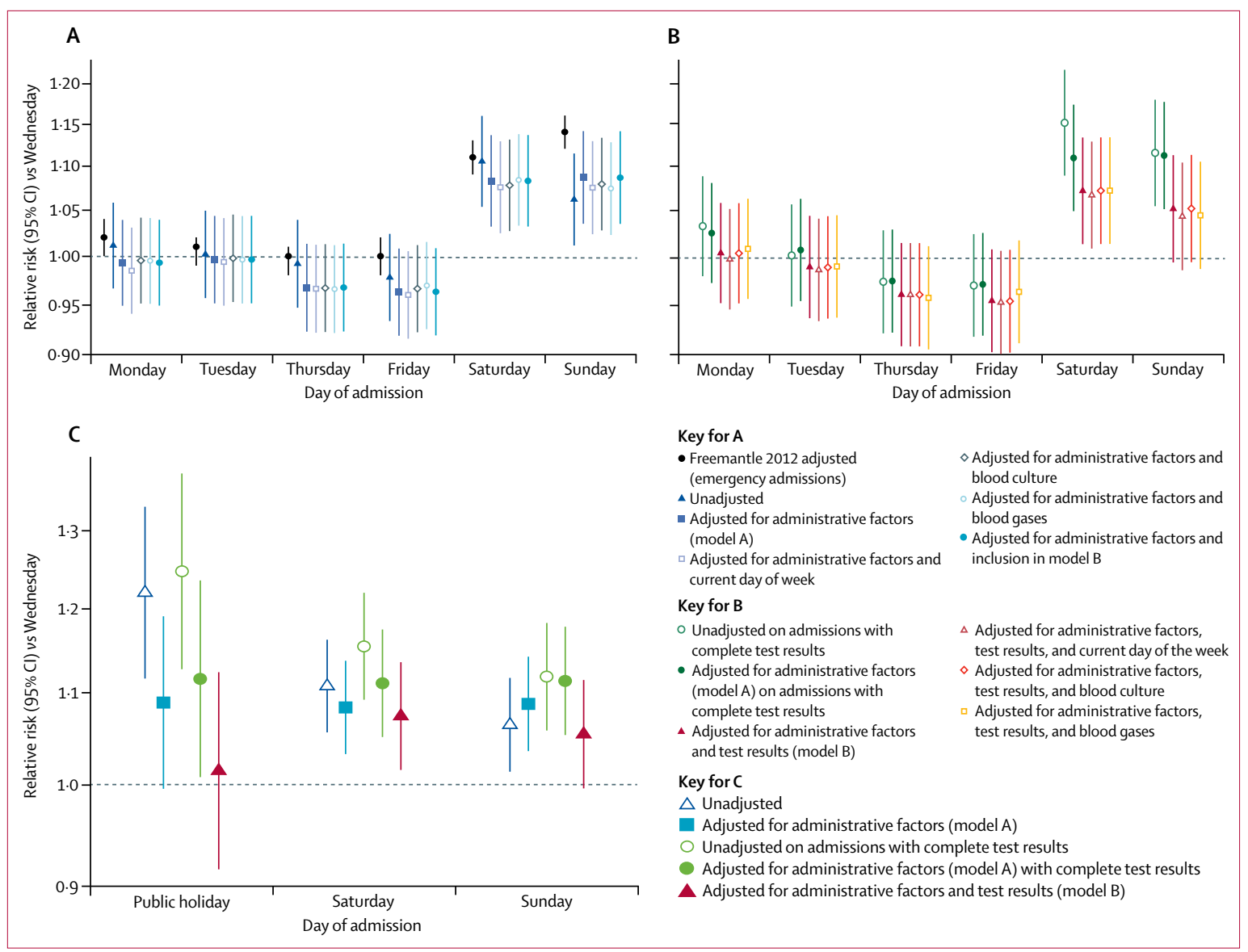

Figure 2: Mortality risk associated with day of admission with and without adjustment for admission test results

(A) Mortality risk by day of week of admission in all emergency admissions. (B) Mortality risk by day of week of admission in emergency admissions with complete test results. (c) Mortality risk by public holiday vs Saturday vs Sunday. Freemantle results are reported to two decimal places, and therefore plotted $95 \%$ Cls are not symmetrical.

except in the middle of the day (1100-1500 h). Between $1100 \mathrm{~h}$ and $1500 \mathrm{~h}$ at weekends, evidence of a 1.18-times increase $(95 \%$ CI $1 \cdot 10-1 \cdot 27)$ in relative mortality risk for emergency admission remained.

\section{Discussion}

Findings from large-scale studies of hospital episode statistics have shown that admission to hospital at weekends is associated with increased risk of death within 30 days compared with admission during the week. $^{1-10,16}$ We used a comprehensive warehouse of electronic health records from four hospitals within a large NHS trust to examine the effect of accounting for illness severity and admission time on this excess risk. Results of common haematology and biochemistry tests, not available in previous studies, ${ }^{3-5,7}$ often differed markedly between weekend and weekday admissions and by admission hour. These test results strongly and independently predicted 30-day mortality. In admissions with complete data, adjustment for these results accounted for $33-52 \%$ of the excess mortality associated with weekend emergency admission. Furthermore, the residual so-called weekend effect was predominantly restricted to the middle of the day on Saturdays and Sundays, with no excess mortality remaining for weekend nights. No patient or clinical subgroups had more or less pronounced residual risk of excess mortality.

Previous findings have been interpreted as suggesting that reduced staffing is a major driver of the excess mortality risk associated with weekend admissions (not necessarily the authors' conclusion ${ }^{7}$ ). However, our measures of hospital workload, reflecting adequacy of staffing levels and service provision relative to each specific day of the week, were not associated with mortality. Further, the excess mortality associated with admission on public holidays was almost completely removed by adjusting for test results reflecting physiological dysregulation on admission. That is, the increased unadjusted risk was mostly explained by increased intrinsic mortality risk in patients who presented to hospital during public holidays. Because staffing levels and services are more similar on public holidays and weekends, it seems unlikely that differences in staffing levels or services alone would explain the excess mortality associated with weekend versus weekday admission. This idea is supported by findings from a 

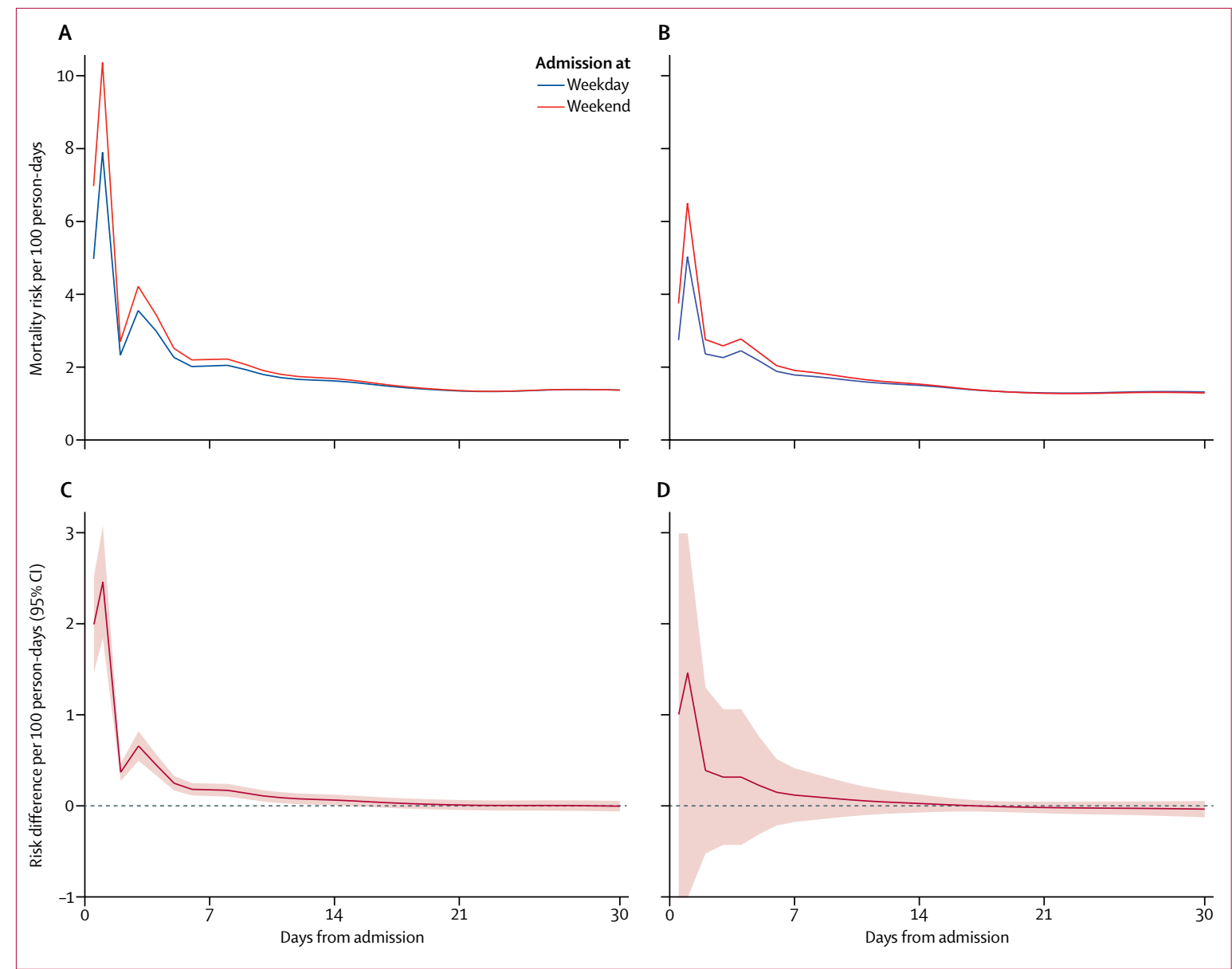

Figure 3: Daily risk of death and excess mortality hazard associated with weekend over weekday admission

(A) Daily risk of death in model A (adjusting for administrative factors in all emergency admissions). (B) Daily risk of death in model B (adjusting for administrative factors and haematology and biochemistry test results). (C) Excess mortality hazard in model A (adjusting for administrative factors in all emergency admissions). (D) Excess mortality hazard in model B (adjusting for administrative factors and haematology and biochemistry test results). 503938 admissions were included in model A; 271465 admissions were included in model B. In each model, absolute mortality risks and excess hazard associated with weekend admission are plotted at the median for all continuous factors, and weighted according to distribution for categorical factors in the relevant model. Risk on the day of admission is estimated at $t=0 \cdot 5$ (ie, at half the day of admission).

previous study ${ }^{11}$ that showed that the weekend effect was unaffected by adjustment for several highly prognostic factors reflecting staffing and service provision in 294602 emergency admissions for specific surgical procedures, and from another study ${ }^{21}$ showing no evidence of association between hospital specialist staffing at weekends and mortality for emergency admissions.

Our data support previous findings ${ }^{7}$ that the main effect is not of current day of the week (Monday-Sunday) the patient is in hospital, only the day of the week of admission. This finding could suggest that effective early management is crucial (the so-called golden hour), supported by the excess mortality risk being greatest in the first 2 days after admission (figure 3). However, failure to manage patients optimally in this golden hour at the weekend (eg, due to reduced staffing or services) could not account for our observation that the weekend effect was restricted to admissions in the middle of the day (1100 $\mathrm{h}$ to $1500 \mathrm{~h}$ ).
Restrictions in access to senior staff, imaging, or other diagnostic tests are similar across the weekend. Other factors we could not account for might explain this mortality excess in the middle of the day. Examples might be reduced access to primary and social care for vulnerable people (eg, at weekends, frail elderly people are more likely to be admitted than referred to more appropriate services), or differences in health-seeking behaviour at weekends (eg, delays in access to primary care, reduced availability of other services, or reluctance to attend emergency departments on Friday or Saturday nights).

Although generally recognised in the literature that the case mix of patients admitted at weekends differs from that of patients admitted on weekdays and that administrative data are inadequate for measurement of illness acuity, these datasets have nevertheless been widely used to investigate the weekend effect. Our ability to investigate the effect of test results, admission time, 

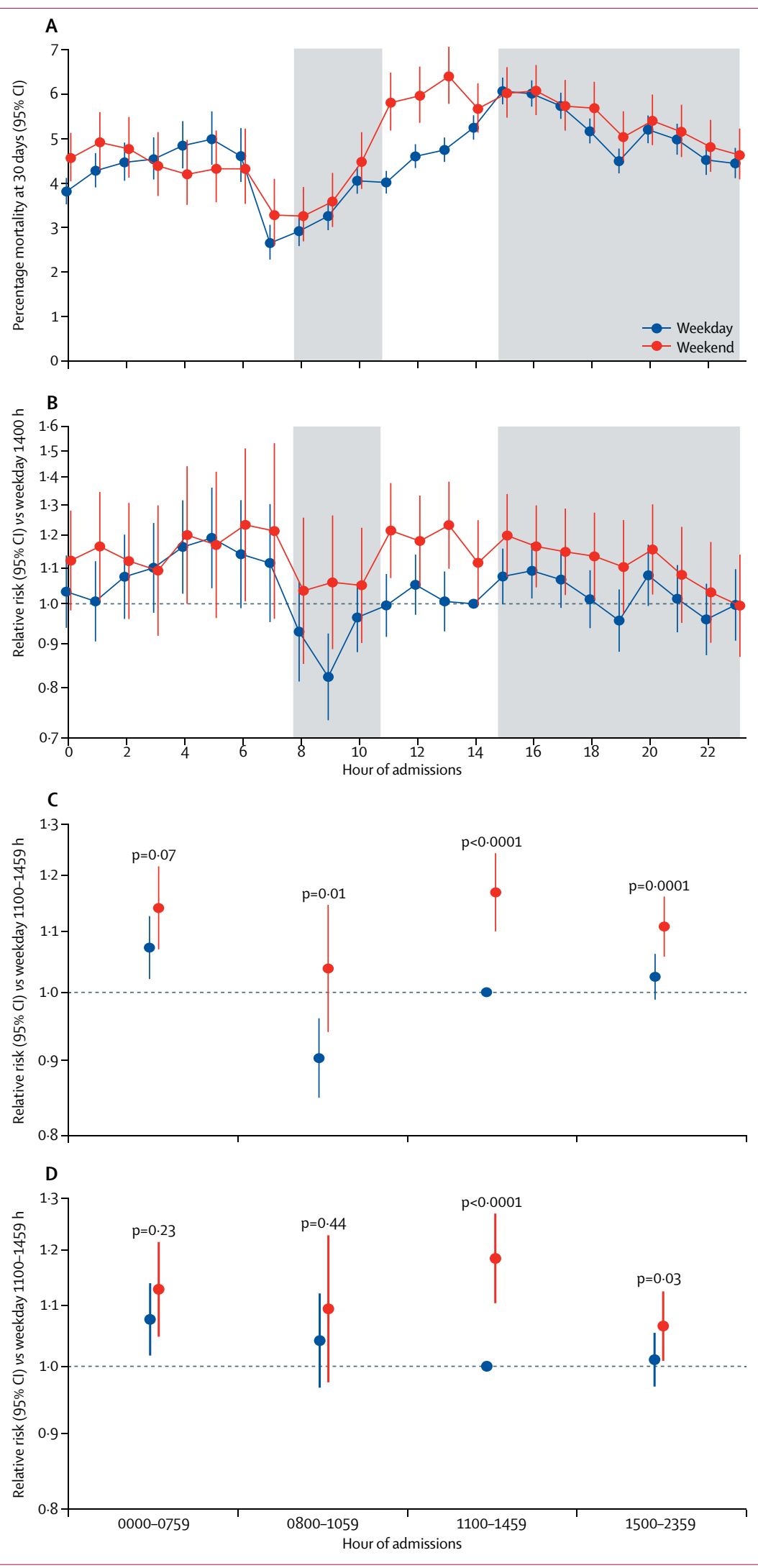

and hospital workload are major strengths of this study. Previous studies have considered overnight versus daytime, or shift patterns in small studies, ${ }^{22}$ in specific conditions, ${ }^{23}$ or did not adjust for illness severity. ${ }^{10}$ We analysed data from unselected emergency admissions, avoiding potential coding bias by restricting our analysis to specific subgroups. ${ }^{24}$ We considered overall mortality because it is most relevant to patients, it incorporates discharge for palliative care, and because being discharged alive is a competing risk for in-hospital mortality. ${ }^{25}$ We did many sensitivity analyses to assess the robustness of our results.

A major limitation is that we did not have access to patients' vital signs or direct measures of acute illness severity (eg, National Early Warning Score, Sequential Organ Failure Score) or frailty (Barthel score). Blood lactate and blood gases were not measured in most patients. In view of the reductions in the weekend effect we found by adjusting for haematology and biochemistry test results, it is plausible that much of the remaining excess risk could be explained by accounting for other patient-level factors. For example, some study findings suggest that adjustment for National Early Warning System score $^{26}$ or arrival by ambulance ${ }^{27}$ (also unavailable in our study) might help to explain the weekend effect. Not all admissions had complete test results; this aspect might limit the generalisability of our model B findings. Completeness was greater in patients who died within 30 days of admission. However, admissions with complete test results were broadly representative of the full dataset (table, appendix p 12), model A provided similar results in all admissions and in admissions with complete test results, and there was no statistical evidence that individuals with complete test results had increased mortality after adjustment. Modest oversampling of more severe cases with more abnormal results, and undersampling of less severe cases with normal results, would (if anything) lead to dilution bias, meaning the genuine effect of test results would be larger, potentially further attenuating the weekend effect. Staffing data were not available, so we used proxies for hospital workload. We might not have found an effect of hospital workload because these were inadequate proxies.

Our study was done in a single hospital group; however, it consists of four large hospitals (including a district general hospital, a large teaching hospital, a specialist orthopaedic hospital, and a major cancer centre), with substantial numbers of emergency admissions to three of the four hospitals, serving a diverse urban and rural local population and accounting for about $1 \%$ of the UK population. Both our hospital mix and the similarity of

Figure 4: 30-day mortality by time and day of admission

(a) Unadjusted 30-day mortality. (B) Model A, including admission hour as a factor. (C) Model A with grouped admission hour. (D) Model B with grouped admission hour. $p$ values for pairwise comparisons of weekend vs weekday admission are shown for parts $C$ and $D$. 
our results to previous multicentre or England-wide studies $^{1,7-8}$ suggest broad generalisability of our findings in terms of the potential for residual confounding from presenting illness severity, with important implications for future studies. For example, investigations of the effect of organisation of health-care services on patient outcomes might be irretrievably biased without adequate adjustment for severity of the presenting illness. Whether the clustering of excess weekend mortality in the middle of the day is generalisable depends on its underlying mechanism, which requires further investigation. Investigators of one large study ${ }^{28}$ of stroke care noted several patterns of weekly variation, probably with different underlying causes. Lastly, standardised mortality ratios, our primary outcome, might not be the most appropriate way to measure quality of hospital care. ${ }^{29}$

In summary, we found that $33-52 \%$ of the residual excess mortality associated with weekend emergency admission after adjustment for standard patient characteristics, and $87 \%$ of the excess mortality associated with emergency admission during public holidays, can be explained by results from 15 commonly measured blood test results. The residual excess is predominantly restricted to weekend admissions between $1100 \mathrm{~h}$ and $1500 \mathrm{~h}$. Mortality is not associated with relative hospital workload. Levels of staffing and services available in hospitals therefore seem unlikely to explain differences in mortality associated with weekend emergency admission. Other more plausible explanations include differences in patients who attend hospitals at these times (eg, in oximetry or vital signs), factors determining their health-care-seeking behaviour, and availability of services outside the hospital at weekends. As such, increased mortality in patients admitted between $1100 \mathrm{~h}$ and $1500 \mathrm{~h}$ at weekends might not be preventable through implementation of 7-day hospital services. ${ }^{1,29}$ By contrast, the unintended consequence of patients delaying presenting to hospital because of fears of worse care at the weekends poses a clear, avoidable danger. ${ }^{30}$

\section{Contributors}

AM planned the statistical analysis, cleaned and analysed the data, and drafted and revised the paper. ASW initiated the project, planned the statistical analysis, cleaned and analysed the data, interpreted the data, and drafted and revised the paper. ASW and AM are the guarantors and their contribution is considered equal. TPQ acquired the data from IORD and revised the paper. NJF advised on medical relevance of test results, interpreted the data, and revised the paper. PW, ML, NS, and DHW interpreted the data and revised the paper. JF monitored data collection and revised the paper. DWC initiated the project, interpreted the data, and revised the paper. TEAP initiated the project, analysed the data, interpreted the data, and revised the paper.

\section{Declaration of interests}

All authors report grants from National Institutes of Health Research (UK), during the conduct of this study. NJF also reports grants from the Medical Research Council UK during the conduct of this study. DHW is employed by Public Health England, part of the UK's Department of Health. ML reports personal fees from Genentech, personal fees from Pfizer, personal fees from Astellas, grants from Merck, and grants from Abbott Diagnostics, outside the submitted work, and has a patent on methods of diagnosis and prognosis in sepsis (14./066.931, pending).

\section{Acknowledgments}

We thank all the people of Oxfordshire who contribute to the IORD, the Research Database Team, and the Patient and Public Panel. We also particularly thank Connie Junghans for comments on the manuscript, and the referees for their thoughtful and constructive reviews and suggestions which have substantially improved the analysis. This work was supported by the National Institute for Health Research Oxford Biomedical Research Centre; an NIHR Research Methods Fellowship to TPQ; and a Medical Research Council UK Clinical Research Training Fellowship to NJF. TEAP and DWC are NIHR Senior Investigators. The views expressed are those of the authors and not necessarily those of the NHS, the NIHR, the Department of Health, or Public Health England. IORD has Research Ethics Committee and Health Research Authority approval as a generic electronic research database (14/SC/1069, ECC5-017[a]/2009).

\section{References}

1 Freemantle N, Richardson M, Wood J, et al. Weekend hospitalization and additional risk of death: an analysis of inpatient data. $J R$ Soc Med 2012; 105: 74-84.

2 Aylin P, Alexandrescu R, Jen MH, Mayer EK, Bottle A. Day of week of procedure and 30 day mortality for elective surgery: retrospective analysis of hospital episode statistics. BMJ 2013; 346: f2424.

3 Aylin P, Yunus A, Bottle A, Majeed A, Bell D. Weekend mortality for emergency admissions: a large, multicentre study. Qual Saf Health Care 2010; 19: 213-17.

4 Ricciardi R, Roberts PL, Read TE, Baxter NN, Marcello PW, Schoetz DJ. Mortality rate after nonelective hospital admission. Arch Surg 2011; 146: 545-51.

5 Barba R, Losa JE, Velasco M, Guijarro C, García de Casasola G, Zapatero A. Mortality among adult patients admitted to the hospital on weekends. Eur J Int Med 2006; 17: 322-24.

6 Handel AE, Patel SV, Skingsley A, Bramley K, Sobieski R, Ramagopalan SV. Weekend admissions as an independent predictor of mortality: an analysis of Scottish hospital admissions. BMJ Open 2012; 2: e001789.

7 Freemantle N, Ray D, McNulty D, et al. Increased mortality associated with weekend hospital admission: a case for expanded seven day services? BMJ 2015; 351: h4596.

8 Ruiz M, Bottle A, Aylin PP. The Global Comparators project: international comparison of 30-day in-hospital mortality by day of the week. BMJ Qual Saf 2015; 24: 492-504.

9 Mohammed MA, Sidhu KS, Rudge G, Stevens AJ. Weekend admission to hospital has a higher risk of death in the elective setting than in the emergency setting: a retrospective database study of national health service hospitals in England BMC Health Serv Res 2012; 12: 87.

10 Lee KG, Indralingam V. A study of weekend and off-hour effect on mortality in a public hospital in Malaysia. Med J Malaysia 2012; 67: 478-82.

11 Ozdemir B, Sinha S, Karthikesalingam A, et al. Mortality of emergency general surgical patients and associations with hospital structures and processes. Br J Anaesth 2016; 116: 54-62.

12 Academy of Medical Royal Colleges. Seven day consultant present care. London: Academy of Medical Royal Colleges, 2012.

13 NHS Services Seven Days a Week Forum. Summary of initial findings. 2013. https://www.england.nhs.uk/wp-content/ uploads /2013/12/forum-summary-report.pdf (accessed Feb 9, 2017).

14 Agency for Healthcare Research and Quality. Classifications software (CCS) for mortality reporting: healthcare cost and utilization project (HCUP). Rockville, MD: Agency for Healthcare Research and Quality, 2017.

15 Meacock R, Anselmi L, Kristensen SR, Doran T, Sutton M. Higher mortality rates amongst emergency patients admitted to hospital at weekends reflect a lower probability of admission.

J Health Serv Res Policy 2016; published online May 6. DOI:10.1177/1355819616649630.

16 Metcalfe D, Perry DC, Bouamra O, et al. Is there a 'weekend effect' in major trauma? Emerg Med J 2016; 33: 836-42.

17 Finney J, Walker A, Peto T, Wyllie DH. An efficient record linkage scheme using graphical analysis for identifier error detection. BMC Med Inform Decis Mak 2011; 11: 7. 
18 Sterne JA, White IR, Carlin JB, et al. Multiple imputation for missing data in epidemiological and clinical research: potential and pitfalls. BMJ 2009; 338: b2393.

19 Freedman LS. Confidence intervals and statistical power of the 'validation' ratio for surrogate or intermediate endpoints. J Stat Plan Inference 2001; 96: 143-53.

20 Royston P, Parmar MK. Flexible parametric proportional-hazards and proportional-odds models for censored survival data, with application to prognostic modelling and estimation of treatment effects. Stat Med 2002; 21: 2175-97.

21 Aldridge C, Bion J, Boyal A, et al, for the HiSLAC Collaborative. Weekend specialist intensity and admission mortality in acute hospital trusts in England: a cross-sectional study. Lancet 2016; 388: 178-86.

22 Maggs F, Mallet M. Mortality in out-of-hours emergency medical admissions-more than just a weekend effect. J R Coll Physicians Edinb 2010; 40: 115-18.

23 Vest-Hansen B, Riis AH, Sørensen HT, Christiansen CF. Out-of-hours and weekend admissions to Danish medical departments: admission rates and 30-day mortality for 20 common medical conditions. BMJ Open 2015; 5: e006731.

24 Li L, Rothwell PM and the Oxford Vascular Study. Biases in detection of apparent "weekend effect" on outcome with administrative coding data: population based study of stroke. BMJ 2016; 353: i2648.
25 Wolkewitz M, Cooper BS, Bonten MJM, Barnett AG

Schumacher M. Interpreting and comparing risks in the presence of competing events. BMJ 2014; 349: g5060.

26 Mohammed MA, Faisal M, Richardson D, et al. Adjusting for illness severity shows there is no difference in patient mortality at weekends or weekdays for emergency medical admissions. QJM 2016; published online July 11. DOI:10.1093/qjmed/hcw104.

27 Anselmi L, Meacock R, Kristensen SR, Doran T, Sutton M. Arrival by ambulance explains variation in mortality by time of admission: retrospective study of admissions to hospital following emergency department attendance in England. BMJ Qual Saf 2016; published online Oct 18. DOI:10.1136/bmjqs-2016-005680.

28 Bray BD, Cloud GC, James MA, et al, for the SSNAP collaboration. Weekly variation in health-care quality by day and time of admission: a nationwide, registry-based, prospective cohort study of acute stroke care. Lancet 2016: 388: 170-77.

29 Hogan H, Zipfel R, Neuburger J, Hutchings A, Darzi A, Black N Avoidability of hospital deaths and association with hospital-wide mortality ratios: retrospective case record review and regression analysis. BMJ 2015; 351: h3239.

30 Gan H-W. Results of the Hunt effect surveys in response to "Increased mortality associated with weekend hospital admission: a case for expanded seven day services?” BMJ 2015; 351: h4596. 